\title{
Rapid Flow Cytometric Method for Measuring Mitochondrial Membrane Potential, Respiratory Burst Activity, and Intracellular Thiols of Human Whole Blood Leukocytes
}

\author{
E. Matteucci ${ }^{*}, 1$, S. Manzini ${ }^{1}$, M. Ghimenti ${ }^{2}$, C. Consani ${ }^{1}$ and O. Giampietro ${ }^{1}$ \\ ${ }^{I}$ Department of Internal Medicine, University of Pisa, Pisa, Italy \\ ${ }^{2}$ Department of Laboratory Medicine and Molecular Diagnostic, University of Pisa, Pisa, Italy
}

\begin{abstract}
Identifying key perturbations of redox regulatory mechanisms may help to understand stress-related disease processes. Currently, flow cytometry allows the functional characterisation of redox changes that occur in different cellular compartments: changes in mitochondrial membrane potential, respiratory burst activity, and intracellular thiol levels are measurable in live white blood cells. However, flow cytometric techniques generally require leukocyte fraction isolation, followed by re-suspension and counting. The purpose of the study was to develop a rapid cytofluorimetric assay to measure the redox triplet in peripheral leukocytes without preliminary isolation.
\end{abstract}

\section{INTRODUCTION}

Redox chemistry is involved in cell signalling and redoxresponsive regulatory mechanisms [1-2]. Mitochondria are considered a cellular signal amplifier capable of generating and conveying Reactive Oxygen Species (ROS) in addition to electrical and calcium signals. Mitochondrial functions have been implicated in the homeostasis of the immune system [3]. Human phagocytic cells of immune system possess an intricate mitochondrial network that is required for cellular processes such as chemotaxis and maintenance of cell shape, respiratory burst activation, and commitment to apoptosis [4].

Identifying key perturbations of redox signalling may help to understand oxidative stress-related disease processes. Currently, useful techniques to assess the activities of living cells, signalling molecules, compartments, or organelles are based on fluorescent probes $[5,6]$. Changes in mitochondrial membrane potential $(\Delta \Psi \mathrm{m})$, ROS generation, and cellular nonprotein thiols can be analysed.

In comparison with the fluorescent antibody techniques, flow cytometric protocols using intracellular fluorochromes are more demanding and hard to standardise. In particular, the measurement of the redox parameters above necessitates the isolation of the leukocytes fraction followed by re-suspension and counting (the cell density needed is usually 1 to $5 \times 10^{5}$ cells $/ \mathrm{mL}$ ). Handling-induced changes in cell parameters cannot be excluded.

Moreover, the concentrations of fluorescent probes and effectors in literature vary over a wide range so to prevent data comparison [6].

The purpose of this study was to develop a rapid and reproducible fluorimetric assay to measure the redox triplet in live human whole blood leukocytes for clinical applications.

*Address correspondence to this author at the Dipartimento di Medicina Interna, via Roma 67, 56126 Pisa, Italy; Tel: +39-050-993246; Fax: +39050-553414; E-mail: ematteuc@int.med.unipi.it
The carbocyanine fluorescent probe 5,5',6,6'-tetra chloro1,1',3,3'-tetraethylbenzimidazolyl-carbocyanine iodide (JC-1) assessed changes in $\Delta \Psi \mathrm{m}$. Cotreatment with the protonophore carbonyl cyanide 3-chlorophenylhydrazone (CCCP) served as a positive control of $\Psi \mathrm{m}$ collapse. Dihydrorhodamine 123 (DHR) was applied as qualitative marker of intracellular ROS. The tumour promotor and activator of protein kinase $\mathrm{C}$ phorbol 12-myristate 13-acetate (PMA) was used as a positive control of oxidative burst. Intracellular thiols were labeled with 5Chloromethyl fluorescein diacetate (CMFDA) [6].

\section{MATERIALS AND METHODOLOGY}

Fluorescent probes were obtained from Invitrogen S.R.L. (Milan, Italy); all other reagents were from Sigma-Aldrich (Milan, Italy).

Ready-to-use stock solutions of JC-1 $(1.06 \mathrm{mmol} / \mathrm{L})$, DHR $(0.26 \mathrm{mmol} / \mathrm{L}), \mathrm{CMFDA}(1.3 \mathrm{mmol} / \mathrm{L})$, and CCCP $(5.3$ $\mathrm{mmol} / \mathrm{L}$ ) were made in dimethyl sulfoxide (DMSO) and stored in aliquots at $-20^{\circ} \mathrm{C}$.

Stock solutions of PMA (1.6 mmol/L in DMSO) were stored in aliquots at $-20^{\circ} \mathrm{C}$; working solutions were made in phosphate buffer solution (PBS) immediately before use.

During analytical method development, venous blood was obtained from 20 adult volunteers, either healthy subjects and patients with diabetes mellitus and/or other chronic inflammatory and autoimmune disorders, in order to cover a wide enough range of redox states; the final methodology was assessed in another group of 20 healthy subjects in order to assess the normal range. Three milliliters of venous whole blood were collected in vacutainer tubes containing ethylenediamine-tetraacetic acid and assayed immediately for fluorescence staining. Baseline blood count was determined using Celldyn 4000 (Abbott Divisione Diagnostici, Roma, Italy). Two microliters of staining solution of each probe were added to $100 \mu \mathrm{L}$ aliquots of whole venous blood. Two microliters of PBS were added to the resting test tubes, whereas $2 \mu \mathrm{L}$ of the effector solutions (CCCP or PMA) were added to 
the stimulated test tubes containing $\mathrm{JC}-1$ or $\mathrm{DHR}$, respectively.

Due to uncertainties in the literature data, scalar final (i.e. during cell incubation) concentrations of the following staining and effector solutions were tested: 5 to $20 \mu \mathrm{mol} / \mathrm{L} \mathrm{JC}-1$, 5 to $50 \mu \mathrm{mol} / \mathrm{L} \mathrm{CMFDA,} 25$ to $100 \mu \mathrm{mol} / \mathrm{L} \mathrm{CCCP,} 0.1$ to 1.0 $\mu \mathrm{mol} / \mathrm{L}$ PMA.

Tubes were incubated for $15 \mathrm{~min}$ at $37^{\circ} \mathrm{C}$. At the end of incubation, $3 \mathrm{~mL}$ of erythrocyte lysing solution $\left(\mathrm{NH}_{4} \mathrm{Cl} 155\right.$ $\left.\mathrm{mmol} / \mathrm{L}, \mathrm{KHCO}_{3} 10 \mathrm{mmol} / \mathrm{L}\right)$ were added to all tubes $(1: 30$ dilution), tubes were vortexed and kept on ice until flow cytometry analysis. Cells were analysed in a FACScalibur (BD Biosciences, Milan, Italy). A total of 10,000 cells per sample were acquired in a dot plot using the data analysis program CELL Quest.

JC-1 fluorescence was analysed on FL1 and FL2 channels for the detection of the dye monomer and J aggregates, respectively. The ratio between red/green fluorescence reflects mitochondrial transmembrane potential, whereas cotreatment with the protonophore CCCP results in decreased JC-1 fluorescence ratio and served as a positive control for disruption of mitochondrial membrane potential. $\triangle \Psi \mathrm{m}$ was expressed as percentage [(FL2 : FL1 - FL2 $2_{\mathrm{CCCP}}$ : $\mathrm{FL}_{\mathrm{CCCP}}$ )/ FL2 : FL1] x 100.

DHR and CMFDA fluorescence was analysed on FL1. The results of ROS production of activated neutrophils are shown as Stimulation Index (SI) values, calculated as the ratio between the mean channel fluorescence intensity of PMA-stimulated cells and the mean channel fluorescence intensity of resting cells.

The data were expressed as the mean fluorescence intensity and statistical analysis was performed on Statview Package (Abacus Concepts, Berkeley, California).

\section{RESULTS}

\section{Mitochondrial Membrane Potential}

A quantitative assessment of $\Delta \Psi \mathrm{m}$ in whole blood leukocytes based on positively charged dyes, such as tetramethylrhodamine ethyl ester (TMRE), was unfeasible because TMRE cannot be used at sufficiently high concentrations due to fluorescence quenching and binding of dye to mitochondrial membranes [5].

The cyanide dye JC-1 appeared suitable for probing mitochondria in whole blood leukocytes for its low toxicity, reasonable solubility, and for providing a qualitative/quantitative method where FL2/FL1 ratio is independent of morphological factors.

So far, $\Delta \Psi \mathrm{m}$ has been analysed in cell lines or human leukocytes isolated from venous blood with $\mathrm{JC}-1$, never in whole blood leukocytes [6].

Flow cytometry analysis of whole blood neutrophil $\Delta \Psi \mathrm{m}$ using JC-1 according to our protocol is shown in Fig. (1) (13 volunteers). Whole blood leukocytes were incubated for 15 min at $37^{\circ} \mathrm{C}$ with scalar concentrations of JC-1 (from 5 to 20 $\mu \mathrm{mol} / \mathrm{L})$ in the absence and in the presence of $100 \mu \mathrm{mol} / \mathrm{L}$ CCCP. In a previous flow cytometry analysis of neutrophil $\triangle \Psi \mathrm{m}$ using TMRE, isolated leukocytes had been incubated for $15 \mathrm{~min}$ at $37^{\circ} \mathrm{C}$ with $200 \mathrm{nmol} / \mathrm{L} \mathrm{TMRE}$ (final concentra- tion in the resting test tubes). Co-treatment with increasing concentrations of the protonophore CCCP (25-50-75-100 $\mu \mathrm{mol} / \mathrm{L}$ in the stimulated test tubes) resulted in dosedependent decreases in mean fluorescence intensity [6]. It had been concluded that $100 \mu \mathrm{mol} / \mathrm{L}$ CCCP was the maximally effective concentration.

Mitochondrion-specific fluorescence could be estimated by CCCP caused collapse of $\Delta \Psi \mathrm{m}$ (cells treated with an excess of $\mathrm{CCCP})$.

$\Delta \psi \mathrm{m}(\%)$

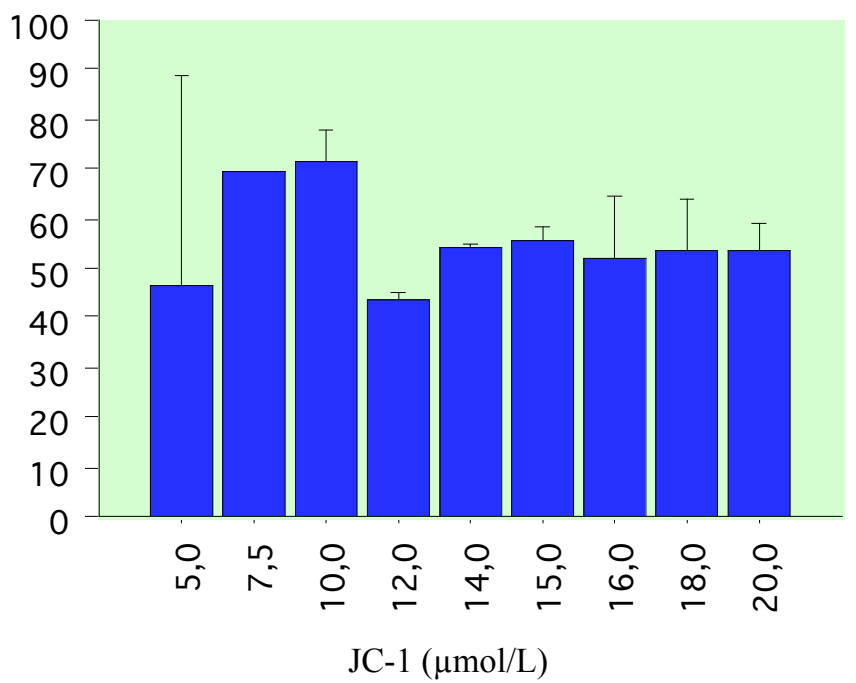

Fig. (1). The cell bar chart shows the dissipation of mitochondrial membrane potential $\left(\Delta \Psi \mathrm{m} \%=\left[\left(\mathrm{FL} 2: \mathrm{FL} 1-\mathrm{FL} 2_{\mathrm{CCCP}}: \mathrm{FL} 1_{\mathrm{CCCP}}\right) /\right.\right.$ FL2 : FL1] x 100) of whole blood neutrophils incubated with increasing concentrations of JC-1 (from 5 to $20 \mu \mathrm{mol} / \mathrm{L}$ ) in the absence and in the presence of the uncoupling agent CCCP (100 $\mu \mathrm{mol} / \mathrm{L})$.

The dissipation of mitochondrial potential induced by CCCP, expressed as percentage of untreated cells, remained stable in the range of $14-20 \mu \mathrm{mol} / \mathrm{L}$ of $\mathrm{JC}-1$ present in the medium. Hence, the mean fluorescence ratio FL2/FL1 was then obtained at a dye concentration of $20 \mu \mathrm{mol} / \mathrm{L}$.

The mean intra-assay coefficient of variation (CV) of 10 duplicates was calculated to be $8.5 \%$. Consistently with previous data [7], in 20 healthy subjects JC-1 FL2/FL1 mean fluorescence ratio was $1.8 \pm 0.9$ (geometric mean 1.7) and decreased to $0.8 \pm 0.2(0.7)$ in the presence of CCCP.

The advantage of this method is the possibility for the simultaneous measurement of $\Delta \Psi \mathrm{m}$ of neutrophils, monocytes, and lymphocytes by gating subpopulations.

\section{Neutrophil Respiratory Burst}

The nonfluorescent probe DHR that, upon the activation of ROS, is converted to rhodamine-123 detected ROS generated by neutrophil NADPH oxidases. The cationic rhodamine localises preferentially within the mitochondria and exhibits green fluorescence. Most cell studies have loaded DHR at concentrations of 5-20 $\mu \mathrm{mol} / \mathrm{L}$ [6]. In our experience, probably because of the stability of the staining, the lowest concentration $(5 \mu \mathrm{mol} / \mathrm{L})$ just obtained a sufficient fluorescent stain even for whole blood assay. 
The forward and side light scatter profiles were adjusted to ensure that the neutrophil population was clearly displayed. Data were then collected from the resting and stimulated tubes. A histogram of rhodamine fluorescence (FL1) was obtained from the gated region and the mean channel fluorescence recorded from the display statistics.

The correlation between the results of the DHR flow cytometry assay (expressed as SI) and increasing concentrations of PMA (from 0.1 to $1.0 \mu \mathrm{mol} / \mathrm{L}$ ) in the stimulated tubes is shown in Fig. (2) (8 volunteers).

There was a significant correlation $(\mathrm{r}=0.87, \mathrm{P}<0.0001)$. The mean intra-assay CV of 10 duplicates was $5.1 \%$. In 20 healthy volunteers, the mean SI was $152 \pm 57$ (geometric mean 141) in agreement with values previously found in leukocytes [8].

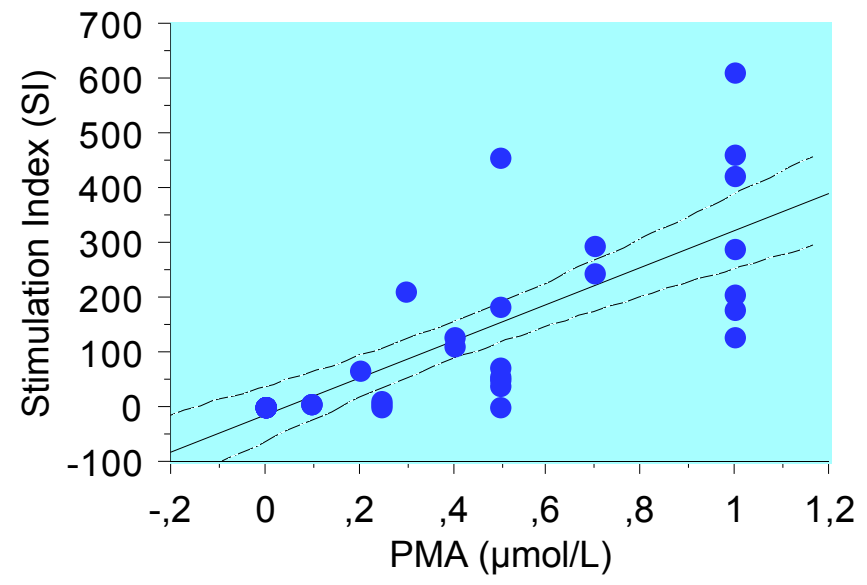

Fig. (2). The scattergram shows the Stimulation Index (SI) plotted against PMA scalar concentrations. SI is the ratio between the mean channel fluorescence intensity of PMA-stimulated cells and the mean channel fluorescence intensity of resting cells

\section{Cellular Thiol Levels}

Changes in the cellular redox environment are considered one of the signalling pathways of the cell, where the principal redox systems are thermodynamically connected to each other. The intracellular thiols are represented by proteinbound $\mathrm{SH}$ groups and free non-protein thiols (predominantly GSH).

The total level of free intracellular thiol was measured using CMFDA, a non-fluorescent probe that has a thiol reactive chloromethyl moiety.

CMFDA freely diffuses through the membrane of live cells. In the cell, esterase mediated hydrolysis converts the probe to the fluorescent 5-chloromethylfluoresceine which forms an impermeant, fluorescent, aldehyde-conjugate product by reacting with thiols on proteins and peptides [6].

In literature, cell staining has been performed at CMFDA concentrations ranging from 1 to $22 \mu \mathrm{mol} / \mathrm{L}$ [5]. In our hand, mean fluorescence intensity increased in a linear manner when whole blood specimens were incubated with increasing concentrations of the probe $(5,25$, and $50 \mu \mathrm{mol} / \mathrm{L})$ (Fig. 3).

Since probe toxicity toward blood cells has not been thoroughly investigated, we chose to use the intermediate concentration for our experiments and incubated whole blood with $25 \mu \mathrm{mol} / \mathrm{L}$ CMFDA.

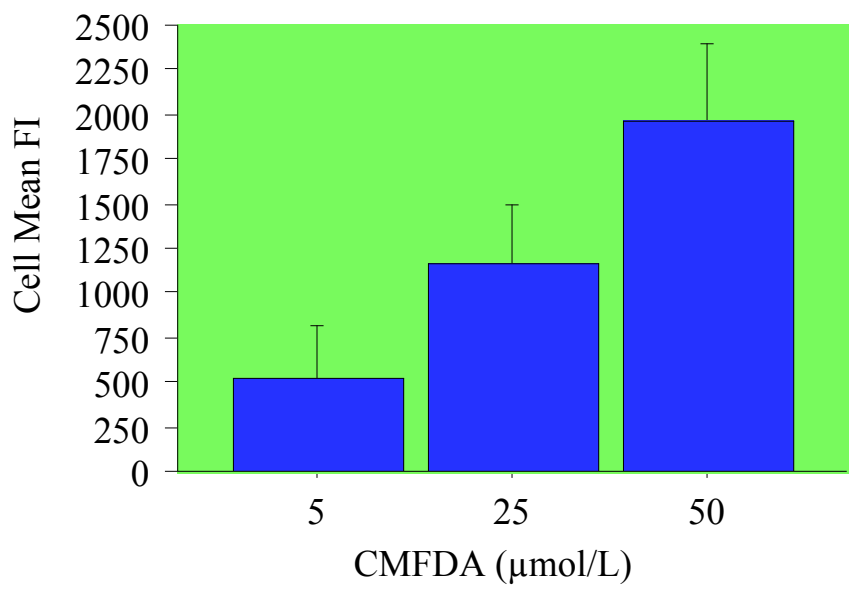

Fig. (3). The effect of increasing concentrations of CMFDA on neutrophil mean fluorescence intensity $(\mathrm{FI})$.

Due to the lack of specific effectors that could be added to the stimulated test tubes, a double determination was made on each sample. The intra-assay $\mathrm{CV}$ of 20 duplicates was $6.1 \%$. In 20 healthy volunteers, mean fluorescence intensity of leukocytes incubated with $25 \mu \mathrm{mol} / \mathrm{L}$ CMFDA was $2158 \pm 527$ (geometric mean 2103).

A comparison with published data [9] must take into account the fact that fluorescence intensity is proportional to dye concentration [10].

\section{DISCUSSION AND CONCLUSION}

The redox triplet composed of the mitochondrial membrane potential, respiratory burst activity, and intracellular thiol levels may be important in several disease states and can be investigated using fluorescent probes. However, existing evaluation methods are laborious, because they are performed on isolated leukocytes, and thus cannot be considered suitable for large-scale clinical investigations. Moreover, sample washing and technical manipulation of the separation procedure have an undoubted impact on the cells that cannot be studied in a physiological environment. Unlike most assays, recent studies included $[11,12]$, the flow cytometry assay described here used whole blood samples. The staining process is quick, cost-efficient, and simple, since it merely involves incubating whole blood in the presence of the dye. The assay system described in this report permits rapid examination of cell redox triplet in volumes of blood as small as $100 \mu \mathrm{L}$, it employs live cells incubated for brief times in their natural environment. There is little technical manipulation required. Furthermore, the DHR and JC-1 assays clearly distinguish between resting and stimulated (with PMA or CCCP) neutrophils. The results obtained with this methodology in whole blood are consistent with literature data, with the specific concerning cellular thiol levels. Indeed, because the thiol dye attains a concentrationdependent staining intensity, inter-laboratory comparisons are only possible by comparing samples incubated with the same dye concentration.

The reproducibility of flow cytometric measurements was assessed by the within-assay coefficient of variation that is a statistical measure of repeat variability. Assay reproducibility can be said good since the CV was less than $10 \%$. Unfortunately, the between-assay repeatibility of fluores- 
cence measurements in live cells cannot be determined by ordinary procedures because redox parameters change during storage.

\section{CONCLUSIONS}

Notwithstanding changes in cell redox triplet are probably involved in a number of disease states, a systematic and large-scale investigation of these parameters using flow cytometry in clinical settings has been unfeasible due to technical drawbacks. Taken together, our experimental data demonstrate that whole blood assay is possible. The ability to measure quickly three redox parameters may provide a useful clue to the mechanisms of disease. We are particularly interested in the origin of the extensive oxidative stress we observed in type 1 diabetes families, non-diabetic relatives included [13]. Oxidative stress in healthy relatives, manifested by protein oxidation and lipid peroxidation, could not be attributed to any evident metabolic disorder. The cytofluorimetric method described here has been developed in order to investigate potential sources of oxidative damage, having taken into account that mitochondrial oxidative metabolism may be the major one.

$$
\begin{aligned}
& \text { ABBREVIATIONS } \\
& \Delta \Psi \mathrm{m}=\text { Mitochondrial membrane potential } \\
& \text { CCCP = Carbonyl cyanide 3-chlorophenylhydrazone } \\
& \text { CMFDA }=5 \text {-Chloromethyl fluorescein diacetate } \\
& \mathrm{CV}=\text { Coefficient of variation } \\
& \text { DHR = Dihydrorhodamine } 123 \\
& \text { DMSO = Dimethyl sulfoxide } \\
& \text { FI } \quad=\text { Fluorescence intensity } \\
& \text { JC-1 = 5,5',6,6'-Tetra chloro-1, } 1^{\prime}, 3,3^{\prime} \text { '-tetraethyl ben- } \\
& \text { zimidazolyl-carbocyanine iodide } \\
& \text { PBS = Phosphate buffer solution } \\
& \text { PMA = Phorbol 12-myristate 13-acetate } \\
& \text { ROS }=\text { Reactive Oxygen Species }
\end{aligned}
$$

SI $\quad=$ Stimulation Index

TMRE = Tetramethylrhodamine ethyl ester

\section{REFERENCES}

[1] Forman, H.J.; Fukuto, J.M.; Miller, T.; Zhang, H.; Rinna, A.; Levy, $\mathrm{S}$. The chemistry of cell signalling by reactive oxygen species and 4-hydroxynonenal. Arch. Biochem. Biophys., 2008, 477, 183-195.

[2] Dröge, W. Free radicals in the physiological control of cell function. Physiol. Rev., 2007, 82, 47-95.

[3] Giovannetti, A.; Pierdominici, M.; Di Iorio, A.; Cianci, R.; Murdaca, G; Puppo, F.; Pandolfi, F.; Paganelli, R. Apoptosis in the homeostasis of the immune system and in human immune mediated diseases. Curr. Pharm. Des., 2008, 14, 253-268.

[4] Fossati, G.; Moulding, D.A.; Spiller, D.G.; Moots, R.J.; White, M.R.; Edwards, S.W. The mitochondrial network of human neutrophils: role in chemotaxis, phagocytosis, respiratory burst activation, and commitment to apoptosis. J. Immunol., 2003, 170, 1964-1972.

[5] Zorov, D.B.; Kobrinsky, E.; Juhaszova, M.; Sollott, S.J. Examining intracellular organelle function using fluorescent probes: from animalcules to quantum dots. Circ. Res., 2004, 95, 239-252.

[6] Matteucci, E.; Giampietro, O. Flow cytometry study of leukocyte function: analytical comparison of methods and their applicability to clinical research. Curr. Med. Chem., 2008, 15, 596-603.

[7] Cossarizza, A.; Ceccarelli, D.; Masini, A. Functional heterogeneity of an isolated mitochondrial population revealed by cytofluorometric analysis at the single organelle level. Exp. Cell. Res., 1996, 222, 84-94.

[8] Wu, Y.-C.; Huang, Y.-F.; Lin, C.-H.; Shieh, C.-C. Detection of defective granulocyte function with flow cytometry in newborn infants. J. Microbiol. Immunol. Infect., 2005, 38, 17-24.

[9] Dietzmann, J.; Thiel, U.; Ansorge, S.; Neumann, K.H.; Täger, M. Thiol-inducing and immunoregulatory effects of flavonoids in peripheral blood mononuclear cells from patients with end-stage diabetic nephropathy. Free Radic. Biol. Med., 2002, 33, 1347-1354.

[10] Chikahisa, L.; Oyama, Y.; Okazaki, E.; Noda, K. Fluorescent estimation of $\mathrm{H}_{2} \mathrm{O}_{2}$-induced changes in cell viability and cellular nonprotein thiol level of dissociated rat thymocytes. Jpn. J. Pharmacol., 1996, 71, 299-305.

[11] Sternfeld, T.; Schmid, M.; Tischleder, A.; Mudra, S.; Schlamp, A.; Kost, B.P.; Gruber, R.; Youle, M.; Bogner, J.R.; Goebel, F.-D. The effect of HIV infection and antiretroviral therapy on the mitochondrial membrane potential of peripheral mononuclear cells. Antivir. Ther., 2007, 12, 769-778.

[12] Cassart, D.; Fett, T.; Sarlet, M.; Baise, E.; Coignoul, F.; Desmecht, D. Flow cytometry probing for mitochondrial function in equine peripheral blood mononuclear cells. BMC Vet. Res., 2007, 3, 25.

[13] Matteucci, E.; Giampietro, O. Oxidative stress in families of type 1 diabetic patients. Diab. Care, 2000, 23, 1182-1186. 